\title{
COMPOSIÇÃO FLORÍSTICA E FITOSSOCIOLOGIA DE PLANTAS ESPONTÂNEAS EM PASTAGENS DO GÊNERO BRACHIARIA (Syn. Urochloa) NO NORDESTE PARAENSE
}

\author{
Anna karoliny Oliveira Lima1; Maria do Socorro Bezerra de Araujo2; Núbia de Fátima Alves Santos³; Márcio \\ Roberto da Silva Melo ${ }^{3}$; Vitor Quintela de Sousa²; Augusto José Silva Pedroso4; Luciana da Silva Borges³; Luis \\ de Souza Freitas ${ }^{3}$. \\ ${ }^{1}$ Graduandos em Agronomia da Universidade Federal Rural da Amazônia (UFRA), Paragominas, Pará, Brasil, \\ oliverannakarol@gmail.com, quintelav@gmail.com \\ ${ }^{1}$ Eng Agr. MSc; Universidade Estadual do Norte Fluminense Darcy Ribeiro, Rio de Janeiro, Brasil, \\ mariasberrita@gmail.com \\ 3Professor (a) Dr. (a) da UFRA, Paragominas, Pará, Brasil, nubia.santos@ufra.edu.br, \\ marcio.melo@ufra.edu.br, luciana.borges@ufra.edu.br, luis.freitas@ufra.edu.br \\ 4Prof. Dr. IFPA, Castanhal, Pará, Brasil, augustopedroso@yahoo.com.br
}

RESUMO: O sucesso de programas de manejo de plantas espontâneas depende do conhecimento das características biológicas e ecológicas das mesmas. Desta forma esta pesquisa tem como objetivo caracterizar a composição florística e fitossociologia em populações de plantas espontâneas em ecossistemas de pastagens cultivadas a mais de dez anos, no município de Ipixuna do Pará, Nordeste Paraense, considerando-se três espécies de gramíneas (Brachiaria (Syn. Urochloa) humidicola, Brachiaria (Syn. Urochloa) brizantha cv. Marandu e Brachiaria (Syn. Urochloa) decumbens cv. Basilisk). Os estudos foram conduzidos no Município de Ipixuna do Pará, região nordeste paraense. Para este estudo foi aplicado o método do quadrado inventário na sequência fez-se a identificação e contagem das plantas, e posteriormente realizou-se a identificação através de literatura especializada e comparações com herbários virtuais. O Índices de similaridade de Sörensen (ISS) foi expresso em porcentagem, sendo máximo (100\%) quando todas as espécies são comuns às duas áreas e mínimo (0\%) quando não há espécies comuns. Constatou-se que a composição florística na pastagem de B. (Syn. Urochloa) humidicola, foram identificados, 10 famílias, 12 gêneros e 12 espécies de plantas espontâneas, em B. (Syn. Urochloa) brizantha cv. Marandu, foram ideficados, 12 famílias, 15 gêneros e 16 espécies, enquanto que no ecossistemas com B. (Syn. Urochloa) decumbens cv. Basilisk, foram encontrados 13 famílias, 24 gêneros e 25 espécies. O maior índice de similaridade foi entre os ecossistemas B. (Syn. Urochloa) humidicola e B. (Syn. Urochloa) brizantha, enquanto que o menor foi entre os ecossistemas B. (Syn. Urochloa) decumbens e B. (Syn. Urochloa) humidicola.

PALAVRA-CHAVE: Comunidade infestante, Ecossistemas, Índice de similaridade. 


\title{
FLORISTIC COMPOSITION AND PHYTOSOCYOLOGY OF SPONTANEOUS PLANTS IN PASTURES OF BRACHIARIA (Syn. Urochloa) IN THE NORTHESTE OF PARÁ STATE
}

\begin{abstract}
The success of programs of management of spontaneous plants depends on the knowledge of their biological and ecological characteristics. The objective of this research was to characterize the floristic composition and phytosociology in spontaneous plant populations in pasture ecosystems cultivated more than ten years, in the municipality of Ipixuna do Pará, Northeast of Paraense, considering three species of grasses (Brachiaria (Syn. Urochloa) humidicola, Brachiaria (Syn. Urochloa) brizantha cv. Marandu and Brachiaria (Syn. Urochloa) decumbens cv. Basilisk). The studies were conducted in the Municipality of Ipixuna do Pará, northeastern region of Para. For this study was applied the square inventory. Afterwards the identification and counting of the plants were carried, and later identification was made through specialized literature and comparisons with virtual herbaria. The Sörensen Similarity Index (ISS) was expressed in percentage, being maximum (100\%) when all species are common to both areas and minimum (0\%) when there are no common species. It was verified that the floristic composition in the pasture of B. (Syn. Urochloa) humidicola, were identified, 10 families, 12 genera and 12 species of spontaneous plants, in B. (Syn. Urochloa) brizantha cv. Marandu, 12 families, 15 genera and 16 species were idealized, while in the ecosystems with B. (Syn. Urochloa) decumbens cv. Basilisk, 13 families, 24 genera and 25 species were found. The highest index of similarity was between the ecosystems B. (Syn. Urochloa) humidicola and B. (Syn. Urochloa) brizantha, while the smaller one was between the ecosystems B. (Syn. Urochloa) decumbens and B. (Syn. Urochloa) humidicola.
\end{abstract}

KEYWORDS: Ecosystems, Index of similarity, Weed community.

\section{COMPOSICIÓN FLORÍSTICA Y FITOSSOCIOLOGÍA DE PLANTAS ESPONTÁNEAS EN PASTAJES DO GÉNERO BRACHIARIA (Syn. Urochloa) EN NORDESTE PARAENSE}

RESUMEN: El éxito de programas de manejo de plantas espontáneas depende del conocimiento de las características biológicas y ecológicas de las mismas. De esta forma esta investigación tiene como objetivo caracterizar la composición florística y fitosociología en poblaciones de plantas espontáneas en ecosistemas de pastos cultivados a más de diez años, en el municipio de Ipixuna do Pará, Nordeste Paraense, considerando tres especies de gramíneas (Brachiaria (Syn. Urochloa) humidicola, Brachiaria (Syn. Urochloa) brizantha cv. Marandu y Brachiaria (Syn. Urochloa) decumbens cv. Basilisk). Los estudios fueron conducidos en el Municipio de Ipixuna do Pará, región nordeste paraense. Para este estudio se aplicó el método del cuadrado inventario. En la secuencia se hizo la identificación y conteo de las plantas, y posteriormente se realizó la identificación a través de literatura 
especializada y comparaciones con herbarios virtuales. El índice de similitud de Sörensen (ISS) fue expresado en porcentaje, siendo máximo (100\%) cuando todas las especies son comunes a las dos áreas y mínimo (0\%) cuando no hay especies comunes. Se constató que la composición florística en el pastoreo de B. (Syn. Urochloa) humidicola, fueron identificados, 10 familias, 12 géneros y 12 especies de plantas espontáneas, en B. (Syn. Urochloa) brizantha cv. Marandu, fueron idealizados, 12 familias, 15 géneros y 16 especies, mientras que en los ecosistemas con B. (Syn. Urochloa) decumbens cv. Basilisk, se encontraron 13 familias, 24 géneros y 25 especies. El mayor índice de semejanza fue entre los ecosistemas B. (Syn. Urochloa) humidicola y B. (Syn. Urochloa) brizantha, mientras que el menor fue entre los ecosistemas B. (Syn. Urochloa) decumbens y B. (Syn. Urochloa) humidicola.

PALABRA CLAVE: Comunidad infestante, Ecosistemas, Índice de similitud.

Estudos da ecologia das plantas espontâneas são importantes para o estabelecimento de métodos eficientes de controle dessas espécies. Esses estudos podem ser feitos através de levantamentos fitossociológicos que forneçam informações para a compreensão da dinâmica das populações dentro da comunidade espontânea de um determinado espaço (COSTA JUNIOR et al., 2011). Assim, a análise fitossociológica tem se destacado na obtenção do conhecimento sobre as populações e a biologia das espécies de plantas espontâneas constituindo uma importante ferramenta no embasamento técnico de recomendações de manejo e tratos culturais para implantação e condução de culturas (SILVA et al., 2010), sem que ocorra uma interferência das plantas espontâneas no desenvolvimento da forragem cultivada na pastagem.

O sucesso de programas de manejo de plantas invasoras depende do conhecimento das características biológicas e ecológicas das mesmas (DIAS-FILHO, 2011. Desta forma, o insucesso de muitas tentativas de controle dessas plantas em áreas de pastagem, está intimamente relacionado a não observação destas características (DIASFILHO, 1990). O que muitas vezes, contribui para a degradação da pastagem.

A degradação de pastagens é uma das principais limitações para a sustentabilidade da atividade pecuária em regiões tropicais e subtropicais, causando 
grandes prejuízos econômicos e ambientais. Cerca de 60 milhões de hectares na Amazônia Brasileira e no Brasil Central estão degradadas ou em processo de degradação (DIAS-FILHO, 2011). Desta forma, para Mota (2010), grande parte das pastagens no Brasil é caracterizada pela degradação, pelo baixo potencial produtivo e, consequentemente, pela baixa capacidade de suporte animal e baixa produção de carne, de leite e de lã. 0 desequilíbrio desses ecossistemas é um processo contínuo, que prejudica toda a cadeia de produção.

Inicialmente a degradação pode ser caracterizada pela simples mudança na composição botânica da pastagem, em decorrência do aumento na proporção de plantas daninhas e da consequente diminuição na proporção de capim. A proliferação de plantas daninhas na pastagem é uma consequência da degradação e não a causa desse processo (DIAS-FILHO, 2011).

Segundo Townsend et al. (2010), a recuperação e intensificação do uso de pastagens cultivadas devem ser preconizadas a fim de reduzir a expansão em áreas de florestas, propiciando benefícios de ordem ecológica (preservação da biodiversidade), econômica (custo de formação de pastagem maior que o de recuperação) e social (necessidade de mão de obra), com vistas à sustentabilidade dos sistemas pastoris no Bioma Amazônia. As estratégias utilizadas para a reabilitação da capacidade produtiva das pastagens buscam interromper o processo de degradação, combatendo-se as causas a ele associadas.

Em pastagens bem estabelecidas, as plantas daninhas possuem baixa capacidade em competir por recursos naturais (nutrientes, luz, água e espaço). Conforme Brighenti e Oliveira (2011) ao longo do tempo, essas espécies desenvolveram características que proporcionam a sobrevivência em ambientes sujeitos aos mais variados tipos e intensidades de limitações ao crescimento e ao desenvolvimento das plantas. Estas características são bastante peculiares e interferem na estratégia de manejo, desta forma, para se manejar bem estas plantas há necessidade de se conhecer tais características (TEIXEIRA; 
FERNANDES, 2011), as quais são chamadas de agressividade. As características de maior relevância são: a habilidade competitiva, a capacidade de produção de propágulos, a desuniformidade do processo germinativo, a capacidade de germinar e emergir a grandes profundidades, a viabilidade dos propágulos em condições desfavoráveis, os mecanismos alternativos de produção, a facilidade de disseminação dos propágulos e o crescimento e desenvolvimento inicial (BRIGHENTI; OLIVEIRA, 2011).

Desta forma, esta pesquisa tem como objetivo caracterizar a composição florística e fitossociologia em populações de plantas espontâneas em ecossistemas de pastagens cultivadas a mais de dez anos, no município de Ipixuna do Pará, Nordeste Paraense, considerando-se três espécies de gramíneas (Brachiaria (Syn. Urochloa) humidicola, Brachiaria (Syn. Urochloa) brizantha cv. Marandu e Brachiaria (Syn. Urochloa) decumbens cv. Basilisk).

O estudo foi conduzido no assentamento PA-Candiru, no lote 88, localizado no Município de Ipixuna do
Pará, região nordeste paraense. O tipo climático é o Am de acordo com a classificação de Köppen, caracterizado por temperatura, umidade relativa do ar e precipitação pluviométrica anual médias de $26{ }^{\circ} \mathrm{C}, \quad 82,5 \%$ e $2.250 \mathrm{~mm}$, respectivamente, (OLIVEIRA et al., 2004), com um período mais chuvoso (janeiro a junho) e outro menos chuvoso (julho a dezembro). Os solos predominantes são do tipo Latossolo amarelo distrófico (EMBRAPA, 1999).

Os levantamentos de campo foram realizados no período chuvoso, de janeiro a abril. Foram utilizados três piquetes, com área total de aproximadamente 6 ha de pastagens formadas a mais de dez anos, nas quais o manejo de plantas espontâneas é feito, normalmente, com roçagens manuais e, raramente, utilizando-se herbicidas. Cada piquete contém uma espécie de gramínea: Brachiaria (Syn. Urochloa) humidicola (quicuio-da-amazônia), Brachiaria (Syn. Urochloa) brizantha cv. Marandu (braquiarão) e Brachiaria (Syn. Urochloa) decumbens cv. Basilisk (braquiarinha).

Para este estudo foi aplicado o método do quadrado inventário ou censo da 
população vegetal que se baseia na utilização de um quadrado de 1,0 m². Em cada piquete foi delimitada uma parcela fixa com dimensões de 100 x 100 m (1 ha), na qual o quadrado foi arremessado ao acaso por 10 vezes, totalizando 30 amostragens $\left(30 \mathrm{~m}^{2}\right)$, sendo 10 amostragens (área útil de $10 \mathrm{~m}^{2}$ ) em cada ecossistema de pastagem. Após lançado aleatoriamente, quadrado foi forçado com as mãos até tocar o chão, efetuou-se então o corte a $5 \mathrm{~cm}$ de altura do solo. $\mathrm{Na}$ sequência fez-se a identificação e contagem das plantas que se encontrarem dentro do quadrado, com as espécies não identificadas foram confeccionadas exsicatas e posteriormente realizou-se a identificação através de literatura especializada e comparações com herbários virtuais. A revisão nomenclatural foi realizada com base em Lorenzi et al. (1976), enquanto que para a classificação taxonômico das familias foi utilizado o sistema Angiosperm Phylogeny Group (APG II, 2003).

O Índices de similaridade de Sörensen (ISS) é expresso em porcentagem, sendo máximo (100\%) quando todas as espécies são comuns às duas áreas e mínimo (0\%) quando não há espécies comuns.

Como resultado, o estudo fitossociológico apresenta: i) relação das espécies inventariadas contendo nome científico, nome vulgar e família botânica e ii) índice de similaridade de Sörensen (ISS).

Utilizou-se o programa Microsoft Office Excel ${ }^{\circledR}$ para realização de análise descritiva e obtenção de tabelas e análise de frequências, referentes à composição florística das comunidades, parâmetros fitossociológicos, padrão de distribuição espacial das espécies e índices de diversidade e equabilidade de espécies.

No presente trabalho, foram encontrados 887 indivíduos, distribuídos em 18 famílias, 33 gêneros e 35 espécies. Aproximadamente $51 \%$ do número de indivíduos pertencem a apenas seis famílias, sendo Fabaceae-Faboideae a mais abundante com 267 indivíduos, seguida de Rubiaceae (129), Violaceae (104), Asteraceae (77), Cyperaceae (75) e Verbenaceae (65). As famílias que apresentaram maior riqueza foi Fabaceae-Faboideae e Poaceae com 5 espécies cada uma, seguida de 
Asteraceae (3), Cyperaceae (3) e Malvaceae (3) (Tabela1).

Ferreira et al. (2014), verificaram em pastagens degradada na região Médio Vale do Rio Doce, Minas Gerais, 23 espécies de plantas espontâneas, distribuídas em dez famílias, sendo oito da família Poaceae, cinco da família Malvaceae e três da família Fabaceae. Galvão et al. (2011), verificaram no levantamento fitossociológico em pastagens em Autazes-AM, que as famílias em maior número de espécies nas áreas foram Poaceae (7) e Cyperaceae (4). As espécies (família) mais comuns encontradas nos ecossistemas amostrados foram: Desmodium incanum (Fabaceae-Faboideae), Lantana camara (Verbenaceae), Mimosa pudica (Fabaceae-Mimosoideae), Odontadenia puncticulosa (Apocynaceae), Scleria melaleuca (Cyperaceae), Serjania laruotteana (Sapindaceae), Solanum palinacanthum (Solanaceae), Spermacoce verticillata (Rubiaceae), Stachytarpheta cayennensis (Verbenaceae) e Vernonia ferruginea (Asteraceae) (Tabela 1). Para Pelissari et al. (2011) grande partes das áreas de pastagens do Brasil é deixada a segundo plano, muitas vezes por poucos investimentos direcionado a pesquisas do controle de plantas daninhas. Segundo Inoue et al. (2013), é importante, que haja, mais estudos controle de plantas espontâneas, com o propósito de indicar as ações para controle das espécies.

A Tabela 2, apresenta uma síntese do quantitativo da composição florística das comunidades de espontâneas amostradas nos ecossistemas de pastagens, onde na pastagem de B. (Syn. Urochloa) humidicola, foram identificados, 10 famílias, 12 gêneros e 12 espécies de plantas espontâneas, em B. (Syn. Urochloa) brizantha cv. Marandu, foram ideficados, 12 famílias, 15 gêneros e 16 espécies, enquanto que nos ecossistemas com B. (Syn. Urochloa) decumbens cv. Basilisk, foram encontrados 13 famílias, 24 gêneros e 25 espécies. Para Mascarenhas et al. (2009), em pastagem degradada de capim-braquiária (U. decumbens), a família Poaceae (Gramineae) apresentou o maior número de espécies, seguida da Leguminosae (Fabaceae) - Mimosoideae. 
Tabela 1. Espécies de plantas espontâneas encontradas em ecossistemas de pastagens de B. (Syn. Urochloa) humidicola, B. (Syn. Urochloa) brizantha Cv. Marandu e B. (Syn. Urochloa) decumbens cv. Basilisk, no município de Ipixuna do Pará, PA.

\begin{tabular}{|c|c|c|}
\hline Família botânica & Nome científico & Nome vulgar \\
\hline Apocynaceae (1) & Odontadenia puncticulosa (Rich.) Pulle & Cipó-cururú \\
\hline \multirow[t]{3}{*}{ Asteraceae (3) } & Vernonia ferruginea Less. & Assa-peixe \\
\hline & Blainvillea biaristata DC. & Picão-grande \\
\hline & Synedrella nodiflora (L.) Gaertn. & Botão-de-ouro \\
\hline Convolvulaceae (1) & Ipomoea asarifolia (Desr.) Roem. \& Schult. & Salsa \\
\hline \multirow[t]{3}{*}{ Cyperaceae(3) } & Cyperus iria L. & Tiririca \\
\hline & Scleria melaleuca Rchb. ex Schltdl & Capa-cachorro \\
\hline & Rhynchospora nervosa (Vahl) Boeck. & Capim-estrela \\
\hline Euphorbiaceae (1) & Jatropha gossypiifolia L. & Pião-roxo \\
\hline \multirow[t]{2}{*}{ Fabaceae-Caesalpinioideae (2) } & $\begin{array}{l}\text { Chamaecrista nictitans subsp. patelaria (DC. Ex Collad.) H. S. } \\
\text { Irwin \& Barneby }\end{array}$ & Falsa-sensitiva \\
\hline & Senna obtusifolia (L.) H.S. Irwin \& Barneby & Fedegoso \\
\hline \multirow[t]{5}{*}{ Fabaceae-Faboideae (5) } & Desmodium barbatum (L.) Benth. & Carrapicho-beiço-de-boi \\
\hline & Glycine wightii (Graham ex Wight \& Arn.) Verdc & Soja-perene \\
\hline & Zornia reticulata Sm. & Urinana \\
\hline & Desmodium incanum DC. & Agarra-agarra \\
\hline & Crotalaria pallida Aiton & Crotalária \\
\hline Fabaceae-Mimosoideae (1) & Mimosa pudica L. & Juquiri-rasteiro \\
\hline Hypericaceae (1) & Vismia guianenses (Aubl.) Choisy & Lacre \\
\hline \multirow[t]{3}{*}{ Malvaceae (3) } & Sida acuta Burm. f. & Guanxuma \\
\hline & Malvastrum coromandelianum (L.) Garcke & Guaxuma \\
\hline & Waltheria indica $\mathrm{L}$. & Guanxuna-branca \\
\hline Orchidaceae (1) & Epidendrum denticulatum Barb. Rodr. & Orquídea \\
\hline Phytolaccaceae (1) & Seguieria langsdorffii Moq. & Limãozinho \\
\hline \multirow[t]{5}{*}{ Poaceae (5) } & Eragrostis plana Nees & Capim-chorão \\
\hline & Chloris gayana Kunth & Capim-pé-de-galinha \\
\hline & Brachiaria (Syn. Urochloa) ruziziensis R. Germ. \& Evrard & Braquiária-peluda \\
\hline & Panicum dichotomiflorum Michx & - \\
\hline & Paspalum virgatum $\mathrm{L}$. & Capim-duro \\
\hline Rubiaceae (1) & Spermacoce verticillata $L$. & Vassourinha-de-botão \\
\hline Sapindaceae (1) & Serjania laruotteana Cambess & Cipó-timbó-açú \\
\hline \multirow[t]{2}{*}{ Solanaceae (2) } & Solanum palinacanthum Dunal & Arrebenta-cavalo \\
\hline & Solanum viarum Dunal & Arrebenta-cavalo \\
\hline \multirow[t]{2}{*}{ Verbenaceae (2) } & Lantana camara L. & Chumbinho \\
\hline & Stachytarpheta cayennensis (Rich.) Vahl & Gervão-azul \\
\hline Violaceae (1) & Hybanthus calceolaria (L.) Oken & Pepaconha \\
\hline
\end{tabular}


Tabela 2. Síntese da composição florística de comunidades de plantas daninhas em pastagens cultivadas, no Município de Ipixuna do Pará, Nordeste Paraense.

\begin{tabular}{lccc}
\hline Pastagem & Famílias & Gêneros & Espécies \\
\cline { 2 - 4 } & & $\mathrm{n}^{\circ} /$ há & \\
\hline B. (Syn. Urochloa) humidicola & 10 & 12 & 12 \\
B. (Syn. Urochloa) brizantha cv. Marandu & 12 & 15 & 16 \\
B. (Syn. Urochloa) decumbens cv. Basilisk. & 13 & 24 & 25 \\
\hline
\end{tabular}

De acordo com os índices de similaridade de Sörensen (ISS), estimados entre os ecossistemas estudados, verificaram-se maiores similaridades entre os ecossistemas B. (Syn. Urochloa) brizantha cv. Marandu e B. (Syn. Urochloa) decumbens cv. Basilisk com 53,66\% e $B$. (Syn. Urochloa) humidicola e B. (Syn. Urochloa) brizantha cv. Marandu com 50\%, demonstrando alta homogeneidade entre os dois ecossistemas (Tabela 3). Ferreira et al. (2014) trabalhando com pastagens degradadas, obtiveram similaridade de 47\%. Enquanto que Lara et al. (2017), obtiveram o índice de similaridade entre os ambientes avaliados elevado, pois seus valores foram superiores a $50 \%$.

O ISS pode ser determinado como elevado quando for superior a 50\%. Tal similaridade pode ser explicada pela proximidade entre ecossistemas analisadas, visto que todos estão localizados numa mesma propriedade, desta forma a alta homogeneidade pode estar relacionada com o(s) sistema(s) de manejo e práticas culturais, utilizadas pelo proprietário e com as características das espécies forrageiras.

Constatou-se que foram encontrados 887 indivíduos, distribuídos em 18 famílias, 33 gêneros e 35 espécies, sendo que a composição florística na pastagem de $B$. (Syn. Urochloa) humidicola, foram identificados, 10 famílias, 12 gêneros e 12 espécies de plantas espontâneas, em $B$. (Syn. Urochloa) brizantha cv. Marandu, foram identificados, 12 famílias, 15 gêneros e 16 espécies, enquanto que nos ecossistemas com B. (Syn. Urochloa) decumbens cv. Basilisk, foram encontrados 13 famílias, 24 gêneros e 25 espécies. 0 maior índice de similaridade foi entre os ecossistemas B. (Syn. Urochloa) humidicola e B. (Syn. Urochloa) brizantha, enquanto que o menor foi entre os 
ecossistemas B. (Syn. Urochloa)

decumbens e B. (Syn. Urochloa)

humidicola.

Tabela 3. Índice de similaridade de Sörensen (ISS) de comunidades de plantas espontâneas ocorrentes em ecossistemas de pastagens de B. (Syn. Urochloa) humidicola, B. (Syn. Urochloa) brizantha cv. Marandu e B. (Syn. Urochloa) decumbens cv. Basilisk, no município de Ipixuna do Pará, PA.

\begin{tabular}{lc}
\hline Comparação & ISS (\%) \\
\hline $\begin{array}{l}\text { B. (Syn. Urochloa) humidicola X B. (Syn. Urochloa) } \\
\text { brizantha }\end{array}$ & 50,00 \\
$\begin{array}{l}\text { B. (Syn. Urochloa) brizantha X B. (Syn. Urochloa) } \\
\text { decumbens }\end{array}$ & 53,66 \\
$\begin{array}{l}\text { B. (Syn. Urochloa) decumbens X B. (Syn. Urochloa) } \\
\text { humidicola }\end{array}$ & 48,65 \\
\hline
\end{tabular}

\section{REFERÊNCIAS}

APG (Angiosperm Phylogeny Group) II. An update of the Angiosperm Phylogeny Group classification for the orders and families of flowering plants: APG $\|$. Botanical Journal of the Linnean Society, $v$. 141, p. 399-436, 2003.

BRIGHENTI, A. M.; OLIVEIRA, M. F. Biologia de Plantas Daninhas. IN: OLIVEIRA JUNIOR, R. S.; CONSTANTIN, J.; INOUE, M. H. (Eds.). Biologia e Manejo de Planta Daninhas. Curitiba, PR: Omnipax, 2011. p.1-36.

COSTA JUNIOR, E. P. B.; SILVA, M. R. M.; ROCHA, L. G. F.; CANTANHEDE, J. D.; LIMA, W. S. G.i SALGADO, S. F. Levantamento da comunidade espontânea da cultura do arroz de terras altas no Estado do Maranhão. Cadernos de Agroecologia, Fortaleza, v. 6, n. 2, p.15, 2011.
DIAS-FILHO, M. B. Degradação de pastagens: processos, causas e estratégias de recuperação. 4 ed.rev., atual. e ampl. ed., Embrapa Amazônia Oriental. BelémPA. 2011. 215p.

DIAS-FILHO, M. B. Plantas invasoras em pastagens cultivadas da Amazônia: estratégias de manejo e controle. Belém: Embrapa-CPATU. 1990. 103p. (EmbrapaCPATU. Documentos, 52).

EMBRAPA. Centro Nacional de Pesquisa de Solos. Sistema Brasileiro de Classificação de Solos. Brasília, 1999. 412p.

FERREIRA, E. A., FERNANDEZ, A. G., SOUZA, C. P., FELIPE, M. A., SANTOS, J. B., SILVA, D.V., e GUIMARÃES, F. A. R. (2014) Levantamento fitossociológico de plantas daninhas em pastagens degradadas do Médio Vale do Rio Doce, Minas Gerais. Revista Ceres, 61(4), 502-510. 
GALVÃO, A.K.L.; SILVA, J.F.; ALBERTINO, S.M.F.; MONTEIRO, G.F.P.; CAVALCANTE, D.P. Levantamento fitossociológico em pastagens de várzea no Estado do Amazonas. Planta Daninha, v.29, n.1, p.6975, 2011.

INOUE, M.H.; ISKIERSKI, D., MENDES, K.F., BEN, R., CONCIANI, P.A., PEREIRA, R.L, DALLACORT, R. Levantamento fitossociológico de plantas daninhas em pastagens no município de Nova OlímpiaMT. Revista Agrarian. Dourados, v.6, n.22, p.376-384, 2013.

LARA, R.O.; PEREIRA, I.M.; FERREIRA, E.A.; PEREIRA, G.A.M.; SILVA, D.V.; SILVA, E.B.; ARAÚJO, F.V.; OLIVEIRA, P.A. Análise de cobertura, levantamento florístico e fitossiológico de uma área em recuperação com topsoil na Serra do Espinhaço, Brasil. Revista Espacios. v. 38, n. 39, 2017.

LORENZI, H.J. Principais ervas daninhas do estado do Paraná. Londrina: IAPAR, 1976. 208 p. ilust. (IAPAR. Boletim Técnico, 2).

MASCARENHAS, M.H.T; VIANA, M.C.M.; LARA, J.F.R.; BOTELHO, W.; FREIRE, F.M.; MACÊDO, G.A.R. Flora infestante em pastagem degradada sob recuperação, pelo sistema de integração lavourapecuária, em região de cerrado. Revista Brasileira de Milho e Sorgo, v. 8, n. 1, p.4155, 2009.

MOTA, V. A. Integração lavoura pecuária - floresta na recuperação de pastagens degradadas no Norte de Minas Gerais. 2009. 112 p. Dissertação (Mestrado em Ciências Agrárias) - Universidade Federal de Minas Gerais, Montes Claros, 2010.
OLIVEIRA, L. L.; FONTINHAS, R. L.; LIMA, A. M. M.; LIMA, R. J. S. Mapas dos parâmetros climatológicos do estado do Pará: umidade, temperatura e insolação, médias anuais. In: Congresso Brasileiro de Meteorologia, 13., 2004, Fortaleza. Anais...Fortaleza: 2004. 7p. Dísponível em: <http://www.cbmet.com/busca.php>.

Acessado em: 06 nov. 2012.

PELISSARI, A.; MENDONÇA, C.G.; LANG, C.R.; BALBINOT JR., A.A. Avanços no controle de plantas daninhas no sistema de integração lavoura-pecuária. Synergismus Scyentifica, v.6, n.2, p.1-17, 2011.

SILVA, H. P.; NEVES, J. M. G.; JUNIOR, D. S. B.; KARAM, D. Levantamento das plantas espontâneas na cultura do girassol. Revista Verde (Mossoró - RN Brasil) v. 5, n. 1, p. 162 - 167 jan./mar., 2010.

TEIXEIRA, S.R.; FERNANDES, A.L.T. Manejo de plantas invasoras em pastagem. Cadernos de Pós-Graduação da FAZU, v. 2, 13p. 2011. Disponível: <http://www.fazu.br/ ojs/index.php/posfazu/article/viewFile/44 3/335> Acesso: 06 nov. 2012.

TOWNSEND, C. R.; COSTA, N. de L.; PEREIRA, R. G. de A.; MENDES, A. M. Resposta de pastagens degradadas de Brachiaria brizantha cV. Marandu a diferentes níveis e frequências de fertilização do solo. EMBRAPA, Porto Velho, 2010. 6p. (Comunicado Técnico, 363). 